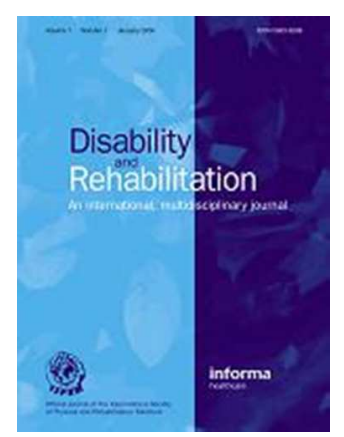

\title{
Engaging stakeholders in rehabilitation research: a scoping review of strategies used in partnerships and evaluation of impacts
}

\begin{tabular}{|r|l|}
\hline Journal: & Disability and Rehabilitation \\
\hline Manuscript ID: & TIDS-04-2014-015.R1 \\
\hline Manuscript Type: & Research Paper \\
\hline Keywords: & $\begin{array}{l}\text { public involvement, disability, knowledge translation, scoping review, } \\
\text { stakeholders, research }\end{array}$ \\
\hline
\end{tabular}

\section{SCHOLARONE}

Manuscripts 
2

Implications for rehabilitation research

- Using several strategies to engage various stakeholders throughout the research process is thought to increase the quality of the research and the rehabilitation process by developing proposals and programs responding better to their needs

- Engagement strategies need to be better reported and evaluated in the literature

- Engagement facilitate uptake of research findings by increasing stakeholders' awareness of the evidence, the resources available and their own ability to act upon a situation

- $\quad$ Factors influencing opportunities for stakeholder engagement need to be better $\underline{\text { understood }}$ 
TITLE

Engaging stakeholders in rehabilitation research: a scoping review of strategies used in partnerships and evaluation of impacts

\author{
AUTHORS \\ Chantal Camden, PT PhD, CanChild Centre for Childhood Disability Research, McMaster \\ University. School of Rehabilitation, Sherbrooke University \\ Keiko Shikako-Thomas, OT PhD, CanChild Centre for Childhood Disability Research, \\ McMaster University \\ Tram Nguyen, CanChild Centre for Childhood Disability Research, McMaster University, School \\ of Rehabilitation Science, McMaster University \\ Emma Graham, BHSc. (Hon.), McMaster University \\ Aliki Thomas, OT PhD, McGill University, School of Physical and Occupational Therapy \\ and Center for Medical Education, Faculty of Medicine, McGill University. Centre for \\ Interdisciplinary Research in Rehabilitation of Greater Montreal (CRIR) \\ Jennifer Sprung, Parent Stakeholder \\ Christopher Morris, MSc DPhil, Senior Research Fellow in Child Health, Peninsula Cerebra \\ Research Unit, Child Health Group, University of Exeter Medical School, Exeter, UK \\ Dianne J. Russell, PhD, CanChild Centre for Childhood Disability Research, McMaster \\ University
}




\section{ABSTRACT}

Purpose:_To describe how stakeholder engagement has been undertaken and evaluated in rehabilitation research.

Method: A scoping review of the scientific literature using five search strategies. Quantitative and qualitative analyses using extracted data. Interpretation of results was iteratively discussed within the team, which included a parent stakeholder.

Results:_Searches identified 101 candidate papers; 28 were read in full to assess eligibility and 19 were included in the review. People with disabilities and their families were more frequently involved compared to other stakeholders. Stakeholders were often involved in planning and evaluating service delivery. A key issue was identifying stakeholders; strategies used to support their involvement included creating committees, organizing meetings, clarifying roles and offering training. Communication, power sharing and resources influenced how stakeholders could be engaged in the research. Perceived outcomes of stakeholder engagement included the creation of partnerships, facilitating the research process and the application of the results, and empowering stakeholders. Stakeholder engagement outcomes were rarely formally evaluated.

\section{Conclusions:}

There is a great interest in rehabilitation to engage stakeholders in the research process. However, further evidence is needed to identify effective strategies for meaningful stakeholder engagement that leads to more useful research that positively impacts practice. 


\section{BACKGROUND}

The knowledge-to-practice-gap in health care and rehabilitation is well documented [1,2].

Traditional knowledge generation and dissemination processes may be one of the crucial reasons for the existence of such a gap [3]. With advances in the science of knowledge translation (KT), the dissemination process is being gradually transformed to include stakeholders (i.e. potential knowledge users such as patients) in the research process. Several reasons for involving stakeholders in the research process have been suggested including: pragmatic (e.g., to facilitate recruitment), theoretical (e.g. to justify the use of a given framework), and mandatory (e.g. requested by funding agency) $[4,5]$. Nevertheless, the most important reason may be that collaborating with stakeholders leads to the identification of more relevant research questions, which results in the creation of knowledge that is more readily transferable, relevant and usable to solving real-world problems $[4,5]$. The assumption is that stakeholder engagement could increase the relevance of research, thereby promoting its use in practice and helping to close the knowledge-to-practice-gap.

In rehabilitation research, authors have called for greater involvement of stakeholders in research $[2,5,6]$. However, no summary of the literature is available to bring together how stakeholder $\underline{\text { engagement in research has been conceptualized, undertaken and evaluated in rehabilitation }}$ research. Summarizing this information would be helpful for designing effective KT partnerships and research proposals. Knowing how best to involve stakeholders could accelerate the uptake and implementation of knowledge to improve interventions, evidence-based practice and policies influencing the research and care for individuals with disabilities. 
The purpose of this paper is to report the findings of a scoping review conducted to identify which stakeholders are involved in rehabilitation research and to describe: (i) effective strategies $\underline{\text { to engage stakeholders meaningfully in the research process, (ii) the factors that influence }}$ engagement, and (iii) the impacts of such engagement. In addition we discuss the implications for researchers and for the field of rehabilitation science.

\section{METHODS}

A scoping review is a review of the literature used to map the key concepts underpinning a research area and the main sources and types of evidence available [7]. To ensure a diversity of perspectives about stakeholders' engagement, efforts were made to include co-authors with different background (e.g., senior researchers, students, a parent, physical and occupational therapy postdoctoral fellows.). A six-step iterative process $[7,8]$ was used to guide the scoping review. We outline the specific steps in the following sections.

\section{Step 1: Identifying the research question(s)}

The questions guiding the scoping review were: "How has stakeholder engagement been conceptualized in rehabilitation research: who are the stakeholders, what strategies are used, what factors influenced engagement and what are the impacts of engagement?".

\section{Step 2: Identifying relevant scientific articles}

Four team members (CC, KST, TN, EG) searched the scientific literature. With guidance from a librarian, an initial search of the electronic databases Medline, Embase, CINAHL and PsycINFO 
using a combination of keywords yielded too many articles not related to stakeholder engagement in the research process (many related to engagement in the rehabilitation process). The search strategy was modified to narrow the scope of the database search to focus on engagement and participation in research, and used a combination of the following keywords: engagement, participatory research, participation, rehabilitation, translational research, knowledge translation, dissemination and knowledge management (see Supplementary File 1 for a list of keywords used). Key terms were adapted to each database. This search led to more targeted results but only a few articles met our criteria. The team then decided to add, sequentially, four more targeted search strategies: 1) a search within the same databases, searching specifically for "integrated knowledge translation" (iKT) and "rehabilitation"; 2) a search in the INVOLVE database (www.invo.org.uk/) (a comprehensive database specifically focusing on patient, caregiver, and public involvement in health research), screening all abstracts and titles for "rehabilitation" or "disability"; 3) a snowball strategy, wherein team members identified relevant articles (team members represent a variety of professional and research backgrounds, countries and stakeholder roles, including physical and occupational therapist, midwife, graduate students and a parent of a child with disabilities); and 4) backwards citation chasing, (i.e. we reviewed the reference lists of the articles included in the previous steps for eligibility using our inclusion criteria).

\section{Step 3: Article selection}

Potentially eligible articles were read in full by two team members; in case of disagreement regarding inclusion, a third team member was consulted until consensus was reached. For final inclusion, the pragmatic decision of including papers published in English between January 2003 and August 2013 was taken since preliminary scanning of the literature allowed us to estimate the interest for stakeholder engagement increased considerably starting about ten years ago. Papers 
also needed to describe strategies used to engage stakeholders in a specific rehabilitation research project. We defined stakeholders as people whose primary job is not directly in research and included the following groups of individuals: children or adults with disabilities (i.e. chronic health conditions, long-term intellectual or physical disabilities), their families and caregivers, individuals representing community groups, policy-makers, and clinicians (e.g., physical therapists, occupational therapists). To be considered a rehabilitation research project, a study had to involve individuals with disabilities or relate to rehabilitation interventions. With regard to engagement, we built on the Canadian Institutes of Health Research (CIHR) definition of integrated KT where each "stage in the research process is an opportunity for significant collaboration with knowledge users, including the development or refinement of the research questions, selection of the methodology, data collection and tools development, selection of outcome measures, interpretation of the findings, crafting of the message and dissemination of the results" [9]. We were looking for papers in which stakeholders were reported to have been included throughout the research process and where at least one concrete example of engagement (e.g. meetings) was described. We included both qualitative and quantitative research studies, and opinion/reflection papers as long as they were describing strategies used in a specific study. We excluded opinion papers presenting general statements on the virtue of stakeholder engagement, as well as book chapters and abstracts for which no full papers were available.

\section{Step 4: Data charting}

A data charting form was developed and piloted with five articles. The form included the following categories of information: type of stakeholders engaged, study objectives, methods, rationale for including stakeholders, specific stakeholder engagement strategies, factors influencing engagement, and impacts associated with engagement. We also documented whether 
the factors influencing stakeholder engagement and the impacts reported in the papers were evaluated. Evaluation was defined as the use of some instrument (e.g. survey, questionnaire, interview) to collect information to document factors influencing engagement and impacts related to engagement. No evaluation was considered to have occurred when authors described the process or discussed possible benefits and pitfalls without empirical data. To ensure validity and consistency of data extraction, four team members independently extracted and recorded data for a subset of articles retained $(\mathrm{n}=5)$ and then met to discuss the charted data. Minor modifications concerning principally the headings (i.e. formulation of the questions guiding the data extraction) and the organization of the charting form were made. The final version of the charting form was agreed upon following this exercise. One team member (TN or EG) then extracted data from all remaining articles, and a second team member (KST or CC) verified the data charted.

Disagreements were rare ( $2 \%$ of the data extracted) and consensus was achieved on the data extracted through discussions and revisions of the original articles.

\section{Steps 5 \& 6: Collating, summarizing, reporting, and Consultation}

$\underline{\text { All team members discussed the data extracted. A numerical analysis (i.e. counting the }}$

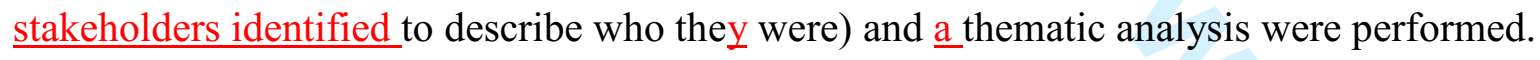
Themes for the thematic analysis related to the aims of the paper (i.e. strategies, factors and impacts) while the subthemes were the concepts nested within each theme. First, four team members met to identify the key concepts emerging from the data charting form. A co-author (KST) extracted all the citations, across references and related the concepts to the subthemes. An iterative process was used to collate the citations, review the data charting form and re-review the original articles when needed to better describe the concepts. In addition, identifying, describing, merging and subdividing the themes was done in collaboration with two other co-authors (CC 
and AT). These three co-authors then discussed the emerging results with the parent stakeholder (JS) to explore the meaning of each theme, provide new perspectives to the interpretations and highlight the most relevant topics for both researchers and stakeholders. All team members reviewed the final results to ensure clarity and consistency.

\section{RESULTS}

The first search strategy identified 74 papers, while the supplementary strategies identified $\underline{a}$ further 27 papers, for a total of 101 papers. Of those, we retained 28 articles based on title and abstracts screening; these articles were read in full to assess eligibility and 19 articles were included (Figure 1). Table 1 outlines the details of the articles included. Individuals with disabilities $(n=13)$ and their families $(n=6)$ were the stakeholders most frequently involved compared to other stakeholders. Other stakeholders included clinicians $(n=9)$, individuals representing community groups $(n=6)$, decision-makers at program and policy levels $(n=2)$ and program managers $(n=1)$. Studies described strategies to involve stakeholders in specific research steps: identifying research questions $(n=10)$, collecting/generating data $(n=14)$, analyzing data $(n=10)$, interpreting results $(n=11)$, disseminating results $(n=11)$, formulating and implementing action plans $(n=9)$. Only one article clearly reported having engaged stakeholders on the writing of the article. No studies evaluated the strategies used. Few studies used data collection to evaluate factors influencing engagement $(n=3)$ or outcomes of engagement $(n=6)$.

[Insert Figure 1 and Table 1 about here] 
Stakeholders were engaged to help identify service users' needs [10-12], to understand the experiences of service users [13-19], to develop and assess the impact of consumer-led programs $[13,20]$, to support the development of strategies and interventions [14, 21], and to identify outcomes that are meaningful for individuals with disabilities [16]. Some articles focused on describing stakeholder engagement in the research process [11, 19, 22-25]. Specifically, articles reflected upon the support needs for engaging individuals who are not researchers [15] and on teamwork between researchers and non-researchers [12].

Approaches presented as useful to support engagement include participatory-action research (PAR) [12, 21, 26], iKT [27], inclusive research [15], community-academic partnerships [28], the Praxis Framework [10], critical/reflexive approaches [18], narrative approaches [20], the Concern report method [10], the PESTEL model [11] and the Radical reflexive approach [18].

\section{Thematic analysis}

\section{Strategies for stakeholder engagement}

\section{Identifying stakeholders}

Two types of strategies were used to recruit stakeholders for volunteer or paid roles: targeted or open. In targeted strategies, researchers selected the organizations or the individuals to be included. Direct invitations were made to partner organizations to nominate members on working committees $[12,20,27]$ or to individuals having previous relationships with the researchers [12]. In open strategies, researchers asked partner organizations to disseminate the invitation to their members (e.g., by mail with a return stamped envelope) or used the media [15, 19, 23, 24]. For some paid positions, researchers drafted a job description delineating stakeholders' roles, with opportunities to renegotiate roles later in the process. No details were provided on the 
interview/selection process. However, many authors suggested paying attention to the following criteria: 1) stakeholders' characteristics (i.e. do they share key features of the group they represent), 2) stakeholders' willingness to speak for the group they represent (as opposed to personal interests), 3) ability to communicate well, 4) achieving diversity in the group [20, 23, 24, 27]. Identifying and engaging the right stakeholders was perceived as a challenge [26]; however partnering with organizations, providing salary and having a clear job description were factors perceived as facilitators for stakeholders' identification and engagement.

\section{Roles and committees}

The creation of one or various committees with different roles (e.g., working, steering or advisory committees, expert panel) was a strategy commonly used [11-13, 16, 19, 26, 28]. Stakeholders included persons with disabilities, caregivers, clinicians, researchers and support/community groups. They were reported to be active and engaged throughout the process. Roles of committee members included: reviewing the proposal and the results [26], being champions of the research program, liaising with research sites and adapting the research accordingly [27]. In some instances, stakeholders were involved in the whole research process, from setting the research agenda and research questions to data collection and analysis, and dissemination $[12,14,19]$. In two papers, stakeholders were considered as co-researchers [19, 23]. Some committees participated in activities such as writing job descriptions, doing interviews and hiring personal, managing funds and organizing social events [13].

Committee activities included face-to-face and teleconference meetings and group discussions $[11,26]$. Frequency and duration of meetings varied across studies but it was perceived to be important to be able to keep stakeholders motivated and engaged. Buettgen et al. [12] reported 
having used face-to-face meetings when decisions needed to be taken, and phone meetings between face-to-face meetings to keep participants informed and engaged. In general, group discussions were held around themes such as service delivery issues, stakeholders' roles and explanation of the research process in general (e.g., explaining the PAR steps to a co-researcher with a disability) $[15,18,19]$. Numbers of participants in those activities varied; some were open to all stakeholders interested while others were done with a smaller, selected group of participants. Written documents (e.g., presenting data to analyze or materials for dissemination) and flipcharts were reported as facilitating participation and were seen as useful to document the stakeholder engagement process $[12,15]$. Other important considerations for engaging stakeholders included scheduling meeting times and locations convenient for stakeholders (e.g., having meetings outside of the service-provider organization), engaging stakeholders in planning agendas, sharing the lead for activities among stakeholders, and outlining a plan for sustainability of group activities from the outset $[12,14,19,26]$.

\section{Supporting stakeholders}

It was perceived that stakeholders needed to be supported in order to understand research and to fulfill their role. Formal training and courses were used to build skills around different research components (e.g., research design, collecting data, facilitating meetings) $[11,12,15,19,25]$ or to increase knowledge on different topics (e.g., client-centred services) [20]. The training occasionally integrated the use of videos to elicit discussions (e.g., about respectful relationships in the research context) [20]. Participants were sometimes paid to attend these training sessions [11]. Informal training was also reported to occur, mostly during committee meetings [18, 19] or during data collection and analysis $[15,17]$. Using a specific framework to interpret data, debriefing field notes and hiring a research assistant as mentor were strategies used to increase 
research skills of the stakeholders. Key strategies found to integrate stakeholders in research are summarized in the Supplementary File 2.

\section{Factors influencing engagement}

Implementation of the strategies described above was perceived to facilitate engagement of stakeholders. In addition, three overarching themes emerged related to factors that positively or negatively influenced stakeholder engagement: communication/culture, power sharing, and resources.

\section{Communication/culture}

Researchers and stakeholders were reported as having different perspectives about stakeholders' roles and expectations. Clarifying and agreeing on realistic expectations at the beginning of the process was recommended to find a balance between obtaining valid research results and meeting personal stakeholders' goal (e.g., having personal information about one's health condition) [13, $14,18,19,23,24]$. This upfront negotiation could avoid conflicts, demotivation, dissolution of partnerships, or frustration in situations where stakeholders could perceive a lack of concrete actions. On the other hand, ongoing communication $[13,18]$, engaging stakeholders in community based activities [27], creating spaces for voicing their concerns [24], and creating risk management strategies (i.e., what to do if problems arise in the group) [13] were perceived to contribute to motivation and engagement, and to foster satisfying partnerships.

Scientific language and research materials (e.g. protocols, pamphlets and questionnaires) needed to be adapted to avoid jargon, ensuring everyone understood and felt comfortable and confident to engage in meaningful dialogue $[11,12,15,16,23,28]$. Since written materials might have low 
meaning for stakeholders with low literacy skills, the use of flip charts and photos might be more appropriate to build on participants' strengths and improve communication [12]. During group discussions, having stakeholders leading conversations could also reduce the cultural barriers, creating a safe environment for the engagement of other participants [19, 27]. However, caution was advised, given that different stakeholder groups (e.g., service users and service providers) can have different expectations. Tensions can arise when one group failed to acknowledge another group's needs and priorities [20]. Facilitators of good group dynamics included planning (in grant proposals) enough time and opportunities to consult and understand the different group needs, to further include their feedback and adapt the materials [24].

\author{
Power sharing \\ Power sharing was essential for establishing a common ground, negotiating the study agenda, \\ resolving conflicts, and supporting meaningful engagement, teamwork and collaboration [10, 12, \\ 20]. The number of stakeholders involved was also a factor to consider for power sharing [13]. \\ Often, few stakeholders represented an entire group and were a minority compared to the number \\ of researchers. Stakeholders, and especially those from vulnerable populations, need to feel \\ entitled to contribute at the same level of the researchers [17]. Researchers' willingness to share \\ control over the research process and their previous experiences with participatory processes \\ were reported as facilitator for stakeholders' engagement [15].
}

Shifting ownership of the research process from researchers to stakeholders takes time [12]. Inviting stakeholders gradually to take more decisions (e.g., setting meeting agendas or taking specific decisions about the research process) was perceived as facilitating power sharing [21, 24]. On the other hand, pre-determined roles and expectations of how stakeholders should participate 
was perceived as a barrier, since it removed the possibility for the stakeholders to determine how they would like to be engaged $[12,14,15,19,23]$. Likewise, engaging stakeholders only at the end of the research project limited power sharing because participants were expected to contribute in a predefined way [16]. Nevertheless, some studies reported having engaged stakeholders exclusively in analysis of the data while providing them with the opportunity to

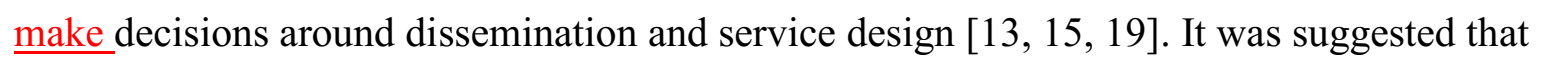
dissemination should ensure stakeholders' opinions are represented [18].

\title{
Time, funding and resources
}

Engaging stakeholders meaningfully required substantial time and financial commitments [11-13, $15,22,23,28]$. Allocating proper financial resources was important to support stakeholders' participation. Costs to consider included traveling expenses, training, support, administration, promotional activities, KT, and accommodating stakeholders' special needs (e.g., adapting materials) $[11,13,15,21,26,27]$. Funding agency deadlines were often perceived as a barrier for creating opportunities for engagement $[11,15,18,22]$. Strategies to overcome time restrictions included hiring staff with time allocated to support stakeholder engagement, and maintaining flexible timelines in the project $[13,14,28]$. Finally, planning for sustainability of stakeholder engagement was key as funds supporting engagement are often not available after data collection and stakeholders have limited opportunities to participate in dissemination activities $[12,15]$.

\section{Impacts related to stakeholder engagement}

\author{
Creating partnerships and building value
}


A frequently mentioned impact was the creation of partnerships where each participant learned to value different perspectives [19, 20, 23]. Researchers can learn about the political system dynamics [27], the potential applications of research findings [23], and the lived experiences of stakeholders [19, 20, 24]. Service users and providers can gain insights on challenges related to service delivery, and immediate applications of research results [11,19]. Partnerships can also evolve into long-term collaborations where other projects can be generated $[12,27]$. Families could also benefit from networking with others through engagement in research [20], and learn about ways of dealing with their members having a disability [22]. Some authors reported that partnerships could promote a model whereby theory, practice and research are interwoven to generate knowledge that will have important benefits for patient care $[10,13]$. This model could lead to significant improvements in the life of persons with disabilities (e.g., increasing accessibility by adding signs and ramps on campus) [11].

\section{Making knowledge more easily applicable and facilitating the research process}

Stakeholders' engagement fostered identification of relevant questions, credibility of the knowledge produced and application of results adapted to contexts [13, 14, 16, 21, 26, 27]. For example, services developed with stakeholders were more widely accepted and responsive to stakeholders' needs [10, 14, 20, 21, 23, 27]. Specifically, engaging policy-makers helped secure funds for new services [27] while engaging individuals from patient support groups facilitated intervention delivery [26]. Engagement also helped adapting the study processes and materials, and facilitated the research process from recruitment to retention and dissemination of results [16, 20-22, 26-28]. Benefits were, however, questioned when stakeholders were consulted only at the end of the project [16]. 


\section{Empowerment}

As a result of engagement, stakeholders increased their confidence and skills, their awareness about specific needs and resources, their ability to advocate, to access information and social support $[12,14,16,19]$. Interactions between stakeholders also contributed to feelings of belonging to a group $[12,20]$. Specifically, PAR contributed to empowerment as stakeholders became more aware of their strengths and personal resources [12, 23]. Authors did mention that stakeholders needed to have real control over how they did their work to ensure engagement would not lead to disempowerment $[15,18]$.

\section{$\underline{\text { Evaluating impacts }}$}

Only six studies collected data to document the impacts of stakeholder engagement $[10,14,15$, $18,19,21]$ and none used standardized measures for evaluation. Evaluations consisted of posthoc analysis of focus groups about stakeholders' engagement [15], debriefing and interviewing stakeholders about their satisfaction with the involvement process [19] and interviews and questionnaires about perceived outcomes around stakeholders' engagement [14]. Qualitative analysis of records around knowledge coproduction in a radical reflective approach was also used as an assessment method [18]. Within PAR, a non-specific reflective approach was used to outline the outcomes of involving stakeholders in relation to the changes in research directions, service delivery and satisfaction with training offered [10, 21].

\section{DISCUSSION}

This scoping review illustrates a diversity of practices associated with stakeholder engagement in rehabilitation research and outlines many potential benefits and challenges in engaging different 
individuals in the entire research process. It also highlights the lack of evidence formally describing and evaluating the engagement in the different stages of research. We identified several factors facilitating and hindering engagement of stakeholders in rehabilitation research. Moreover, this scoping review revealed the complexity of searching for evidence in a body of literature that is still in its infancy and with a large variation in terms and definitions used to describe stakeholder engagement. Our findings and recommendations to move the field forward are presented around key questions about stakeholder engagement in rehabilitation research.

\section{Who's involved and who should be involved?}

Individuals with disabilities and their caregivers were more frequently engaged in rehabilitation research compared to other stakeholder groups. This is not surprising given the emphasis on patient and family-centered care in rehabilitation [29]. Moreover, many of the articles retrieved were about service delivery where it is common to engage direct service users, but other stakeholders are less often represented [30]. We would argue that many other stakeholders groups could also contribute through their unique perspectives, skills and resources. The implication is that researchers should identify their goals up front_and then identify all the stakeholder groups that could be interested or need to be involved to increase project feasibility, outcomes and sustainability. These stakeholders could include decision-makers, health care professionals, administrative personnel, community group representatives as well as researchers in other fields out of the rehabilitation specialties (e.g., politics).

How are stakeholders engaged and when should they be engaged?

Practices around stakeholder engagement identified in this review varied. Many studies reported having engaged stakeholders throughout the research process, but in only a few articles were we 
able to identify specific strategies in each research step (i.e., from identifying research questions to disseminating findings and implementing plans). This could be explained by authors not reporting all the strategies they used, or because stakeholders were informed throughout the process without real opportunities to influence and engage in the process. Using approaches to support stakeholder engagement, such as PAR [26] could help planning for engagement strategies through the research process. Other approaches, such as scholarships of practice (a collaborative model whereby theory, research and practice are interwoven [31]), could also support engagement.

In many studies, stakeholders joined the project once it had already started and were thus not engaged in generating research questions. Collaboration in identifying the research questions is crucial as it impacts the whole process, and influences the ownership over the project $[4,5]$. Opportunities for researchers and stakeholders to interact might need to be in place first to allow stakeholders to participate meaningfully in this step (as opposed to only providing letters of support). Examples of research projects that involve stakeholders in developing consensus on research priorities exist (e.g., [33]); however, they have been funded as a single project. Research funding opportunities might need to be restructured to support involvement in research more broadly, supporting continuous stakeholder involvement in and across projects as opposed to funding engagement in single projects [32].

Data collection and analysis require specialized skills, which stakeholders might not have; this may explain why stakeholders were not always involved in this step. Nevertheless, it is important to identify their desired level of involvement and support it. Involving stakeholders in 
interpretation might be more feasible and could facilitate tailoring knowledge to context, an important step toward generating knowledge that will be useful for practice $[17,34]$.

Many groups have recommended including stakeholders in dissemination and KT $[9,35,36]$. Few articles retrieved reported concrete strategies used in the dissemination and action plan steps. Moreover, the majority of papers were written by researchers who shared their perceptions about the processes and outcomes of stakeholder engagement, without inputs by stakeholders or data about stakeholder engagement. Stakeholders might not have the interest or skills to write scientific papers. Nevertheless, opportunities should be offered to stakeholders to participate in disseminating results. Besides scientific articles, other research outputs might better match stakeholders' skills, comfort level and interests, such as clinical and policy briefs, lay summaries, newsletters and dissemination on social media.

\section{What are the facilitators and barriers to engagement?}

Several barriers and facilitators for stakeholder engagement were identified in the different studies. Factors facilitating integration of stakeholders (e.g. having regular meetings, assigning clear roles, sharing power, and having the time and financial resources) are similar to the ones described in the KT literature. It is also likely that strategies documented as being effective for KT, such as using active and multi-modal approaches, using plain language and fostering continuous interactions between researchers and non researchers $[2,3,37,38]$ would also support stakeholders' engagement through the process. Likewise, barriers to KT such as limited resources could also be barriers for stakeholder engagement. Both funding agencies and researchers need to be aware of the time and resources needed to support engagement. Organizations use different strategies to promote stakeholder involvement, including developing resources (e.g., guidelines 
for stakeholder engagement), coercion (e.g., "obligating" researchers to have letters of support from knowledge users) or targeted grants (e.g., planning grants that allow time to involve stakeholders).

What is the evidence, and what evidence is needed?

We originally intended to include only papers in which strategies for engagement had been trialed and evaluated in rehabilitation research, but we broadened our criteria since no such study was found. Of the articles retained, few used empirical data collection to identify factors influencing engagement or outcomes of engagement. Among these, no standardized measures were used, and the questionnaires, focus group guides and debriefing techniques used were not clearly described. This is consistent with the fact that research documenting stakeholder engagement in the research process is emerging slowly; the mechanisms to ascertain and measure engagement are largely unstructured [39, 40].

An evidence-based approach to promote stakeholder engagement is necessary, where the outcomes are measured and benchmarked to establish the most effective strategies. This would follow the development of KT science in other areas where randomized control trials and observational studies are used to measure the effectiveness of KT interventions [41-44]. Strategies and tools to assess the impacts of stakeholder engagement (e.g., (piiaf.org.uk/), evidence library and database of projects involving stakeholders (e.g., www.involve.org.uk/), and models that could support stakeholder engagement (e.g., the Knowledge-to-Action [34]) are available. However, none of these resources were tested in the articles retrieved. Rehabilitation research needs to move beyond the conceptualization of stakeholder engagement to the use and evaluation of these strategies and models. 


\section{Why is it so difficult to find current evidence?}

Our search strategies were limited by the variety of terms referring to stakeholder engagement and we may have missed relevant articles. Engagement might have many synonyms (e.g., involvement or participation), and can refer to participation as research participants or participation in the rehabilitation process, in community life or in the research process. The word 'stakeholders' may have many synonyms (e.g. partners) and can also be named by "who they are" (e.g., patients, decision-makers). Challenges around having multiple names to label the same concepts, or using the same name to describe different processes, have already been identified for the term "knowledge translation" [45]. All these nuances in language affect the ability to really understand the processes used and the ability to retrieve relevant information.

The parent stakeholder in our team (JS) also pointed out that current evidence about stakeholder engagement might not be in scientific articles but on the Internet and social media, where much discussion is going on. These conversations were, however, not captured by this scoping review, since we only searched evidence in scientific articles to describe how stakeholder engagement was conceptualized in the literature. However, social media could be a mechanism to retrieve, review and evaluate information including a broader and more democratic representation of stakeholder engagement. Social media discussions are not usually critically appraised, but they are increasingly being used by stakeholders to retrieve and disseminate information $[46,47]$ and are gaining a growing appreciation in rehabilitation research and practice [48]. They could also offer new venues to foster stakeholder engagement and contribute to diminishing cultural barriers, fostering understanding of stakeholders' priorities and policy trends, creating effective evaluation 
methods, improving the efficacy of research collaborations and reaching individuals outside traditional circles of evidence-based information.

The parent stakeholder on our team also highlighted that it is currently difficult for stakeholders to be aware of the evidence and the current research opportunities. Researchers might need to actively solicit and build relationships with stakeholders. National databases using the Internet and social media to connect stakeholders and researchers with similar interests could be helpful in building those relationships, presenting clearly the research processes and the expectations around stakeholder involvement. Stakeholders may feel voiceless and limited by shortcomings within the system; being involved in research that leads to intervention and system improvements can be empowering, especially for those who may feel an overwhelming sense of disempowerment.

\section{$\underline{\text { STUDY LIMITATIONS AND FUTURE DIRECTIONS }}$}

As stated above, search strategies were limited by the great variety of terms used for each of the terms searched (e.g., knowledge translation, stakeholders), and the heterogeneity of the field of $\underline{\text { rehabilitation itself (different populations and settings). As a result, relevant articles may have not }}$ been included in our review. Whilst a parent of a child with a disability was part of the team, given our predominant personal experiences in childhood rehabilitation, there are other $\underline{\text { stakeholders across various rehabilitation contexts that were not consulted and whose }}$ perspectives would have been useful to engage. It would be optimal to involve representatives of other stakeholder groups, such as policymakers, decision-makers, clinicians and individuals with various disabilities and ages. 
Homogenizing terms used in rehabilitation research, and defining them consistently will be essential for further developing the field, and contributing to more comprehensive literature $\underline{\text { reviews. The development of solid and long-term relationships with a variety of stakeholders will }}$ $\underline{\text { also improve their participation in research and consequent impacts. }}$

\section{CONCLUSION}

This scoping review revealed that: a) a limited group of stakeholders are being involved in rehabilitation research; b) engagement practices vary; c) the research process is still mainly controlled by the researchers and stakeholders are rarely meaningfully involved in all the research steps; d) barriers and facilitators for engaging stakeholders in research are similar to the ones in KT (e.g., financial and time constraints, culture and language); and e) there have been few evaluations of stakeholder engagement processes and impacts. There is a need to document and evaluate the diversity of approaches and strategies used to integrate stakeholders. This will allow us to better understand how to develop fruitful partnerships between researchers and stakeholders and to quantify the impacts. Identifying what works best under which circumstances is crucial, since it is unlikely that one approach fits all contexts, research goals and stakeholder needs. $\underline{\text { Identifying effective strategies to enable meaningful stakeholder engagement is likely to lead to }}$ research that actually changes practice and improves care.

\section{ACKNOWLEDGEMENT}

We wish to thank Peter Rosenbaum for his critical reviewing of this manuscript, as well as all our colleagues and the stakeholders with whom we have worked with to expand our knowledge around stakeholder engagement in research. 
DECLARATION OF INTEREST

The authors report no declarations of interest. The two first authors receive post-doctoral funding support through the Fonds de Recherche du Québec - Santé (FRQ-S). The first author is also funded by the Canadian Institutes of Health Research (CIHR) and the Canadian Child Health Clinician Scientist Program (CCHCSP).

\section{REFERENCES}

[1] Straus SE, Tetroe J, Graham I. Defining knowledge translation. Can Med Assoc J 2009;181(34):165-8

[2] Menon A, Korner-Bitensky N, Kastner M, McKibbon K, Straus S. Strategies for rehabilitation professionals to move evidence-based knowledge into practice: A systematic review. J Rehabil Med 2009;41(13):1024-32.

[3] Grimshaw JM, Eccles MP, Lavis JN, Hill SJ, Squires JE. Knowledge translation of research findings. Implement Sci 2012;7(50).

[4] Bowen SJ, Graham ID. From knowledge translation to engaged scholarship: Promoting research relevance and utilization. Arch Phys Med Rehabil 2012; 94(1): S3-S8.

[5] Morris C, Shilling V, McHugh C, Wyatt K. Why it is crucial to involve families in all stages of childhood disability research. Devel Med Child Neurol 2011;53(8):769-71.

[6] Rosenbaum P. Family-centred research: What does it mean and can we do it? Devel Med Child Neurol 2011;53(2):99-100.

[7] Arksey H, O'Malley L. Scoping studies: Towards a methodological framework. Int J Soc Res Methodol 2005;8(1):19-32. 
[8] Levac D, Colquhoun H, O’Brien KK. Scoping studies: Advancing the methodology. Implement Sci 2010;5(1):1-9.

[9] Canadian Institutes of Health Research. Guide to knowledge translation planning at CIHR: Integrated and end-of-grant approaches. Ottawa: CIHR; 2012.

[10] Suarez-Balcazar Y, Martinez LI, Casas-Byots C. A participatory action research approach for identifying health service needs of hispanic immigrants: Implications for occupational therapy. Occup Ther Health Care 2005;19(1-2):145-63.

[11] Wood M. Disability, participation and welfare to work in staffordshire. Int J Integr Care $2003 ; 11(2): 43-8$.

[12] Buettgen A, Richardson J, Beckham K, Richardson K, Ward M, Riemer M. We did it together: A participatory action research study on poverty and disability. Disabil Soc 2012;27(5):603-16.

[13] Ottmann G, Laragy C, Damonze G. Consumer participation in designing community based consumer-directed disability care: Lessons from a participatory action research-inspired project. Systemic Practice and Action Research 2009;22(1):31-44.

[14] Suarez-Balcazar Y. Empowerment and participatory evaluation of a community health intervention: Implications for occupational therapy. OTJR 2005;25(4):133-142.

[15] Bigby C, Frawley P. Reflections on doing inclusive research in the "Making life good in the community" study. J Intellect Dev Disabil 2010;35(2):53-61.

[16] Amtmann D, Cook KF, Johnson KL, Cella D. The PROMIS initiative: Involvement of rehabilitation stakeholders in development and examples of applications in rehabilitation research. Arch Phys Med Rehabil 2011;92(10):S12-9.

[17] Cotterell P. Exploring the value of service user involvement in data analysis:'Our interpretation is about what lies below the surface'. Educational Action Research 2008;16(1):5-17. 
[18] Gillard S, Simons L, Turner K, Lucock M, Edwards C. Patient and public involvement in the coproduction of knowledge reflection on the analysis of qualitative data in a mental health study. Qual Health Res 2012;22(8):1126-37.

[19] Walmsley J, Mannan H. Parents as co - researchers: A participatory action research initiative involving parents of people with intellectual disabilities in ireland. British Journal of Learning Disabilities 2009;37(4):271-6.

[20] Sax P. Finding common ground: Parents speak out about family-centered practices. Journal of Systemic Therapies 2007;26(3):72-90.

[21] Taylor RR, Braveman B, Hammel J. Developing and evaluating community-based services through participatory action research: Two case examples. Am J Occup Ther 2004;58(1):73-82.

[22] Hutton E, Coxon K. Involving parents as service users in an interprofessional research project. J Interprof Care 2008;22(6):661-3.

[23] Johns T, Miller P, Kopp K, Carter Z, Cooper G, Johnston J, Nicholas Z, Wright N. Tracey johns research policy and planning equal lives? disabled people evaluate an independent living strategy for essex social services. Journal of Policy and Planning 2004;22(2):51-8.

[24] Williams V, Simons K. More researching together: The role of nondisabled researchers in working with people first members1. British Journal of Learning Disabilities 2005;33(1):6-14.

[25] Walmsley J. Involving users with learning difficulties in health improvement: Lessons from inclusive learning disability research. Nurs Inq 2004;11(1):54-64.

[26] Ehde DM, Wegener ST, Williams RM, Ephraim PL, Stevenson JE, Isenberg PJ, MacKenzie EJ. Developing, testing, and sustaining rehabilitation interventions via participatory action research. Arch Phys Med Rehabil 2013;94(1):S30-42. 
[27] McGrath PJ, Lingley-Pottie P, Emberly DJ, Thurston C, McLean C. Integrated knowledge translation in mental health: Family help as an example. J Can Acad Child Adolesc Psychiatry 2009;18(1):30-7.

[28] Langston AL, Mccallum M, Campbell MK, Robertson C, Ralston SH. An integrated approach to consumer representation and involvement in a multicentre randomized controlled trial. Clin Trials 2005;2(1):80-7.

[29] Bamm EL, Rosenbaum P. Family-centered theory: Origins, development, barriers, and supports to implementation in rehabilitation medicine. Arch Phys Med Rehabil 2008;89(8):1618-24.

[30] Fudge N, Wolfe CD, McKevitt C. Assessing the promise of user involvement in health service development: Ethnographic study. BMJ 2008 Feb 9;336(7639):313-7.

[31] Kielhofner G. A scholarship of practice: Creating discourse between theory, research and practice. Occup Ther Health Care 2005;19(1-2):7-16.

[32] Smits PA, Denis J. How research funding agencies support science integration into policy and practice: An international overview. Implementation Sci 2014;9(1):28.

[33] McIntyre S, Novak I, Cusick A. Consensus research priorities for cerebral palsy: A delphi survey of consumers, researchers, and clinicians. Devel Med Child Neurol 2010;52(3):270-75.

[34] Graham ID, Logan J, Harrison MB, Straus SE, Tetroe J, Caswell W, Robinson N. Lost in knowledge translation: Time for a map? J Contin Educ Health Prof 2006 2006;26(1):13-24.

[35] Armstrong R, Waters E, Dobbins M, Anderson L, Moore L, Petticrew M, Clark R, Pettman TL, Burns C, Moodie M, Conning R, Swinburn B. Knowledge translation strategies to improve the use of evidence in public health decision making in local government: Intervention design and implementation plan. Implement Sci 2013;8(121)..

[36] Turner K. Involve: Briefing notes for researchers : Public involvement in NHS, public health and social care research. UK: Involve; 2012. 
[37] Ketelaar M, Russell DJ, Gorter JW. The challenge of moving evidence-based measures into clinical practice: Lessons in knowledge translation. Phys Occup Ther Pediatr 2008;28(2):191206.

[38] Zidarov D, Thomas A, Poissant L. Knowledge translation in physical therapy: From theory to practice. Disabil Rehabil 2013;35(18):1571-77.

[39] Chaudoir SR, Dugan AG, Barr CH. Measuring factors affecting implementation of health innovations: A systematic review of structural, organizational, provider, patient, and innovation level measures. Implementation Sci 2013;8:22.

[40] Beresford P. The role of service user research in generating knowledge-based health and social care: From conflict to contribution. Evid Policy 2007;3(3):329-41.

[41] Menon A, Cafaro T, Loncaric D, Moore J, Vivona A, Wynands E, Korner - Bitensky N. Creation and validation of the PERFECT: A critical incident tool for evaluating change in the practices of health professionals. J Eval Clin Pract 2010;16(6):1170-75.

[42] Lavis JN, Oxman AD, Moynihan R, Paulsen EJ. Evidence-informed health policy 1-Synthesis of findings from a multi-method study of organizations that support the use of research evidence. Implementation Sci 2008;3(53):7.

[43] Lavis JN, Wilson MG, Grimshaw JM, Haynes RB, Hanna S, Raina P, Gruen R, Ouimet M. Effects of an evidence service on health-system policy makers' use of research evidence: A protocol for a randomised controlled trial. Implement Sci 2011;6:51.

[44] Russell DJ, Rivard LM, Walter SD, Rosenbaum PL, Roxborough L, Cameron D, Darrah J, Bartlett DJ, Hanna SE, Avery LM. Using knowledge brokers to facilitate the uptake of pediatric measurement tools into clinical practice: A before-after intervention study. Implementation Sci $2010 ; 5(1)$ 
[45] McKibbon KA, Lokker C, Wilczynski NL, Ciliska D, Dobbins M, Davis DA, Haynes RB, Straus SE. A cross-sectional study of the number and frequency of terms used to refer to knowledge translation in a body of health literature in 2006: A tower of babel. Implement Sci 2010;5(1).

[46] Grindrod K, Forgione A, Tsuyuki RT, Gavura S, Giustini D. Pharmacy 2.0: A scoping review of social media use in pharmacy. Res Social Adm Pharm 2014;10(1):256-70.

[47] Hamm MP, Chisholm A, Shulhan J, Milne A, Scott SD, Given LM, Hartling L. Social media use among patients and caregivers: A scoping review. BMJ Open 2013;3(5).

[48] Miller ET. How social media affects our practice. Rehabilitation Nursing 2013;38(6):273-74. 
Figure 1. Illustration of the search process to identify articles pertaining to stakeholders' engagement in rehabilitation research

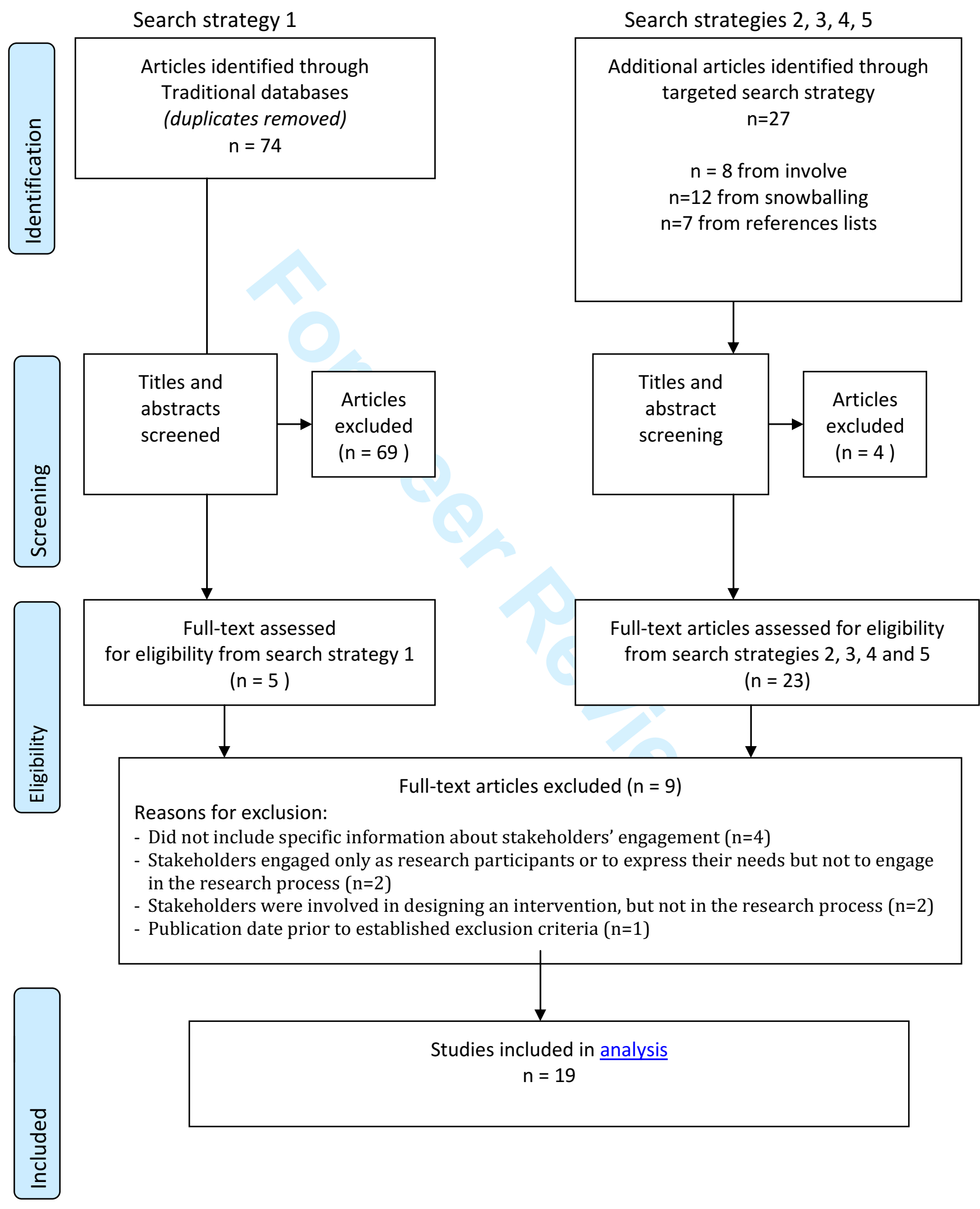

URL: http:/mc.manuscriptcentral.com/dandr Email: davemuller@suffolk.ac.uk 
Table 1: Characteristics of studies retained

\begin{tabular}{|c|c|c|c|c|c|c|c|c|c|c|c|c|c|c|c|c|c|c|c|c|c|}
\hline $\begin{array}{l}6 \\
7 \\
7 \\
8\end{array}$ & First Author & Year & Country & Title & & $\begin{array}{l}\text { e of } S \\
\text { aged }\end{array}$ & Stake & holde & & & $\begin{array}{l}\text { Rest } \\
\text { stak }\end{array}$ & $\begin{array}{l}\text { earch } \\
\text { rehol }\end{array}$ & $\begin{array}{l}\text { steps } \\
\text { ders } u\end{array}$ & $\begin{array}{l}\text { sin } w \\
\text { vere }\end{array}$ & $\begin{array}{l}\text { hich } \\
\text { engag }\end{array}$ & & \begin{tabular}{|l} 
Factors \\
influen \\
engage
\end{tabular} & $\begin{array}{l}\text { cing } \\
\text { ment }\end{array}$ & $\begin{array}{l}\text { Outcc } \\
\text { engag }\end{array}$ & $\begin{array}{l}\text { omes of } \\
\text { gement }\end{array}$ & 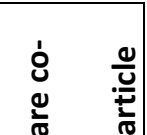 \\
\hline $\begin{array}{l}10 \\
11 \\
12 \\
13 \\
14\end{array}$ & & & & & $\sum_{0}^{0}$ & 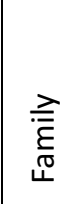 & 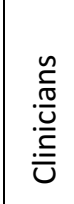 & $\stackrel{\sim}{\dot{\varepsilon}}$ & 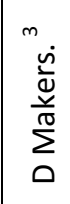 & 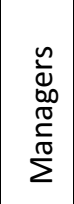 & $\begin{array}{l}\dot{\sigma} \\
\dot{y} \\
\stackrel{0}{\sigma} \\
\dot{\sigma} \\
\dot{0}\end{array}$ & $\begin{array}{l}n \\
\dot{0} \\
0 \\
0 \\
0 \\
0 \\
0\end{array}$ & $\mid \begin{array}{c}\frac{n}{n} \\
\frac{2}{20} \\
\frac{\pi}{4}\end{array}$ & 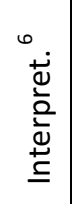 & 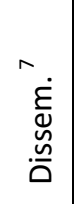 & 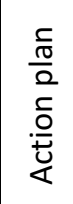 & 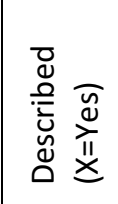 & 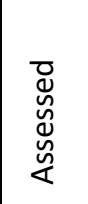 & 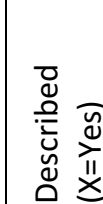 & 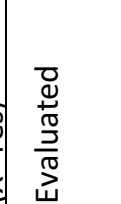 & 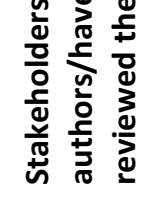 \\
\hline $\begin{array}{l}151 \\
16(16) \\
17 \\
18 \\
19\end{array}$ & Amtmann & 2011 & USA & $\begin{array}{l}\text { The PROMIS Initiative: } \\
\text { Involvement of Rehabilitation } \\
\text { Stakeholders in Development } \\
\text { and Examples of Applications in } \\
\text { Rehabilitation Research }\end{array}$ & $\mathrm{x}$ & & $x$ & & & & $x$ & & & & & & & & $x$ & & \\
\hline $\begin{array}{l}202 \\
21^{2}(15) \\
22\end{array}$ & Bigby & 2010 & Australia & $\begin{array}{l}\text { Reflections on doing inclusive } \\
\text { research in the "Making Life } \\
\text { Good in the Community" study }\end{array}$ & $x$ & & & & & & & $x$ & & & $x$ & & $x$ & $x$ & $x$ & $x$ & \\
\hline $\begin{array}{l}23 \\
24^{3} \\
25^{(12)} \\
26\end{array}$ & Buettgen & 2012 & Canada & $\begin{array}{l}\text { We did it together: a } \\
\text { participatory action research } \\
\text { study on poverty and disability }\end{array}$ & $x$ & & & & & & $x$ & $\mathrm{x}$ & $x$ & $x$ & $x$ & $x$ & & & & & $x$ \\
\hline $\begin{array}{l}274 \\
28(17) \\
29 \\
30 \\
31\end{array}$ & Cotterell & 2007 & UK & $\begin{array}{l}\text { Exploring the value of service } \\
\text { user involvement in data } \\
\text { analysis: 'Our interpretation is } \\
\text { about what lies below the } \\
\text { surface' }\end{array}$ & $x$ & & & & 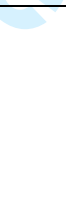 & & $x$ & $x$ & $x$ & $x$ & & & & & & & \\
\hline $\begin{array}{l}325 \\
33(26) \\
34 \\
35\end{array}$ & Ehde & 2013 & USA & $\begin{array}{l}\text { Developing, Testing, and } \\
\text { Sustaining Rehabilitation } \\
\text { Interventions Via Participatory } \\
\text { Action Research }\end{array}$ & $\mathrm{X}$ & & & & & & $x$ & & & & $x$ & $x$ & $x$ & & $\mathrm{X}$ & & \\
\hline $\begin{array}{l}366 \\
37(18) \\
38 \\
39 \\
40\end{array}$ & Gillard & 2012 & UK & $\begin{array}{l}\text { Patient and Public Involvement } \\
\text { in the Coproduction of } \\
\text { Knowledge: Reflection on the } \\
\text { Analysis of Qualitative Data in a } \\
\text { Mental Health Study }\end{array}$ & $x$ & $x$ & $x$ & & & $x$ & $\mathrm{x}$ & $x$ & $x$ & $x$ & & & $x$ & $x$ & $x$ & $x$ & \\
\hline $\begin{array}{l}47 \\
427\end{array}$ & Hutton & 2008 & UK & Involving parents as service & $\mathrm{x}$ & $\mathrm{x}$ & $\mathrm{x}$ & & & & & $x$ & & & & & $x$ & & $x$ & & \\
\hline
\end{tabular}




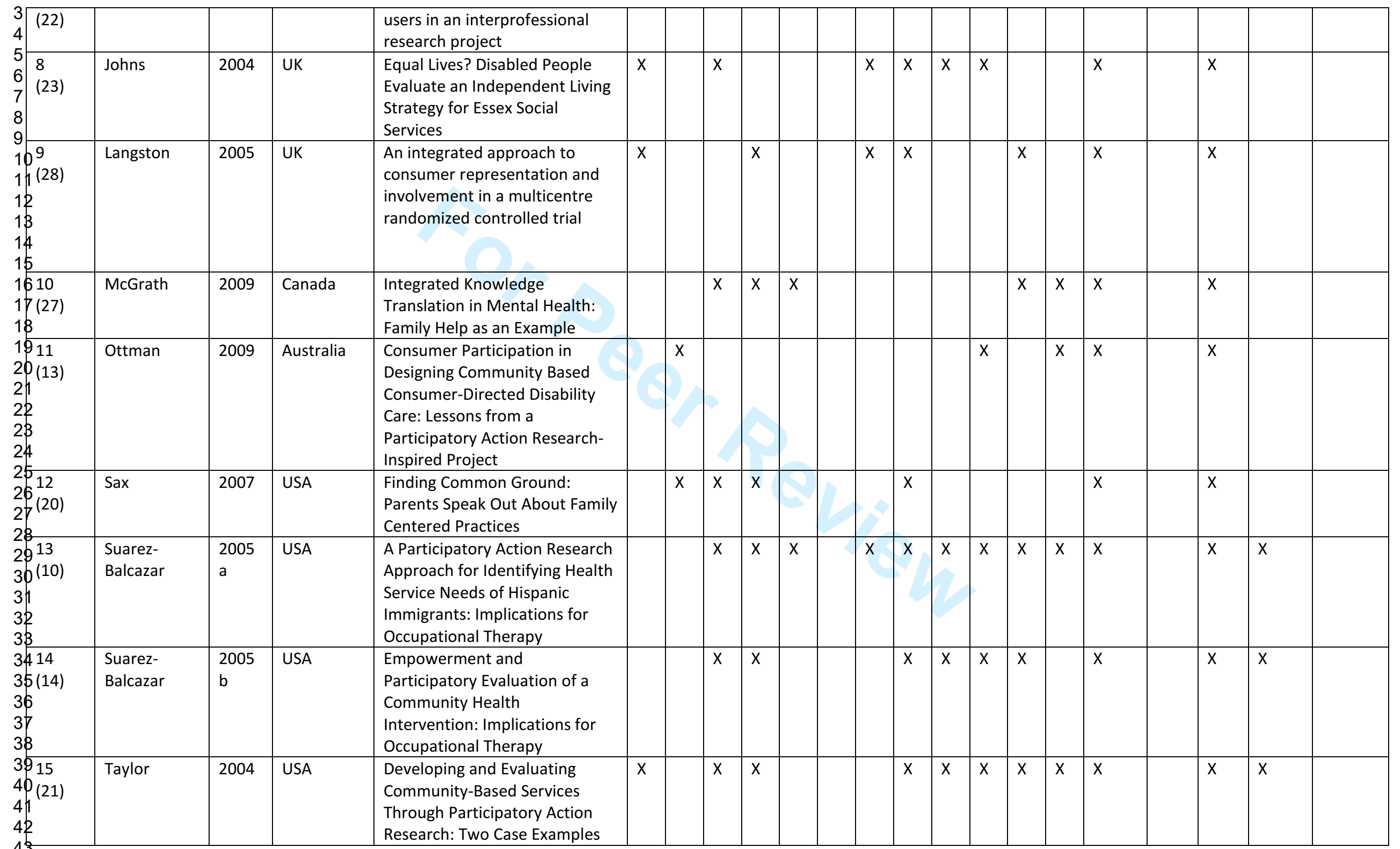




\begin{tabular}{l|l|l|l|l|l|l|l|l|l|l|l|l|l|l|l|l|l|l|l|l|l|l|}
3 & 16 \\
4 & Walmsley & 2009 & Ireland & & $\begin{array}{l}\text { Parents as co-researchers: a } \\
\text { participatory action research } \\
\text { initiative involving parents of } \\
\text { people with intellectual } \\
\text { disabilities in Ireland }\end{array}$ \\
7 \\
8
\end{tabular}

Legend.

1. $P W D=$ Persons with Disabilities

2. Com $=$ individuals representing community groups

3. D. Makers $=$ Decision Makers

4. Id. Quest. = Identification of Research Questions

5. Data Col. $=$ Data Collection/generation

6. Interpret. = Interpretation

Dissemin. $=$ Dissemination of results

Ref \# = Reference number of the article in the reference list at the end of the paper 


\section{Supplemental file 1.}

Keywords used initially that led to many articles which were not related to stakeholder engagement in the research process.

There were four main types of terms that we needed to identify: those relating to knowledge translation, those relating to service providers, those pertaining knowledge users and those relating to decision making and collaboration. Like terms were combined using OR and all four concepts were combined using AND.

The keywords were adapted according to each database (ie MEDLINE, EMBASE, CINAHL, PsycINFO); however, the search strategy for EMBASE is listed below:

Terms relating to knowledge translation

1. translational strateg*.mp. [mp=title, abstract, subject headings, heading word, drug trade name, original title, device manufacturer, drug manufacturer, device trade name, keyword] or

2. exp Translational Medical Research/

or

3. translational research.mp.

or

4. exp "Diffusion of Innovation"/

or

5. implementation science.mp.

or

6. exp Information Dissemination/

or

7. information dissemination.mp.

or

8. exp "Diffusion of Innovation"/

or

9. diffusion of innovation.mp.

AND

Terms relating to service providers

10. occupational therap*.mp. [mp=title, abstract, subject headings, heading word, drug trade name, original title, device manufacturer, drug manufacturer, device trade name, keyword] or 11. physio*.mp. [mp=title, abstract, subject headings, heading word, drug trade name, original title, device manufacturer, drug manufacturer, device trade name, keyword]

or

12. physical therap*.mp. [mp=title, abstract, subject headings, heading word, drug trade name, original title, device manufacturer, drug manufacturer, device trade name, keyword] or 
13. speech language patholog*.mp. [ $\mathrm{mp}=$ title, abstract, subject headings, heading word, drug trade name, original title, device manufacturer, drug manufacturer, device trade name, keyword]

or

14. pediatric*.mp. [mp=title, abstract, subject headings, heading word, drug trade name, original title, device manufacturer, drug manufacturer, device trade name, keyword] or

15. audiolog*.mp. [mp=title, abstract, subject headings, heading word, drug trade name, original title, device manufacturer, drug manufacturer, device trade name, keyword] or

16. Rehabilitation/

or

17. exp Physical Therapy Modalities/

or

18. exp Physical Therapy Specialty/

or

19. rehabilitation.mp.

or

20. rehabilitation*.mp. [mp=title, abstract, subject headings, heading word, drug trade name, original title, device manufacturer, drug manufacturer, device trade name, keyword]

AND

Terms relating to knowledge users

21. exp Family/

or

22. child*.mp. [mp=title, abstract, subject headings, heading word, drug trade name, original title, device manufacturer, drug manufacturer, device trade name, keyword]

or

23. exp Administrative Personnel/

or

24. exp Adolescent/

or

25. youth.mp.

or

26. occupational therapist.mp.

or

27. exp Physical Therapists/

or

28. physiotherapist.mp.

or

29. speech language pathologist.mp.

or

30. pediatrician.mp. 
or

31. audiologist.mp.

or

32. exp Patients/

or

33. patient.mp.

or

34. client.mp.

or

35. stakeholder.mp.

\section{AND}

Terms relating to decision making and collaboration

36. exp Decision Making/

or

37. exp Cooperative Behavior/

or

38. $\exp$ Communication/

\section{AND}

39. limit to (english language and yr="2003 -Current")

The initial search did not effectively address the research question, particularly with respect to engaging stakeholders. Therefore, researchers decided to conduct a new search strategy by choosing keywords from target papers that effectively addressed the research question.

Engagement, participatory research and participation were combined using OR. Rehabilitation, rehabilitation medicine and rehabilitation nursing were also combined using OR. Translational research, translational medical research, knowledge translation, information dissemination and knowledge management were combined using OR. The three concepts were combined using AND, and the list of articles was limited to English language and those published from 2003 onwards. The revised search strategy for EMBASE is below:

1. engagement.mp.

or

2. exp participatory research/

or

3. participation.mp.

AND 
4. exp rehabilitation/

or

5. exp rehabilitation medicine/

or

6. exp rehabilitation nursing/

or

7. rehabilitation.mp.

AND

8. exp translational research/

or

9. translational medical research.mp.

or

12. knowledge translation.mp.

or

13. exp information dissemination/

or

14. exp knowledge management/

AND

16. limit to (english language and yr="2003 -Current") 
Supplemental file 2: Strategies, theoretical approaches and methods, and objectives for engaging stakeholders

Cognitive interviews (Amtman et al., 2011)

Focus/structured groups (Buettgen et al., 2012; Gillard et al., 2012; Walmsley et al., 2009)

Workshops (Dawn et al., 2013)

Regular teleconferences and/or in-person meetings (Buettgen et al., 2012; Dawn et al., 2013; Gillard et al., 2012; Walmsley et al., 2009; Williams et al., 2005; Suarez-Balcazar et al., 2005a)

Sporadic meeting in specific steps of the research process at the stakeholder's locations (e.g school, community center) (Hutton et al., 2008)

Brainstorming sessions about outcomes and possible applications (Suarez-Balcazar et al., 2005b) Service users developed and conducted semi structured interviews (Gillard et al., 2012; Sax et al., 2007)

Stakeholders helped to design project's web page-members and provided feedback (SuarezBalcazar et al., 2005b)

Stakeholders paid as co-researchers (Bigby et al., 2010; Dawn et al., 2013; Williams et al., 2005; Wood et al., 2003)

Stakeholders participating in steering/advisory committee (Buettgen et al., 2012; Dawn et al., 2013; McGrath et al., 2009; Langston et al., 2005; Ottman et al., 2008; Suarez-Balcazar et al., 2005a; Taylor et al., 2004; Walmsley et al., 2009)

Training stakeholders to engage in research: formal and informal training (Williams et al., 2005; Wood et al., 2003)

Use of different media and materials to ascertain engagement and understanding: lay summaries, flipchart, videos, drafts of project at different stages (Bigby et al., 2010; Dawn et al., 2013; Sax et al., 2007; Walmsley et al., 2009; Williams et al., 2005) 